\title{
Endometrioid Stromal and Related Neoplasms
}

National Cancer Institute

\section{Source}

National Cancer Institute. Endometrioid Stromal and Related Neoplasms. NCI Thesaurus. Code C8384.

A category of mesenchymal gynecologic neoplasms. It includes endometrial stromal nodule, endometrioid stromal sarcoma, and undifferentiated sarcoma. Endometrial stromal nodule has been described in the uterine corpus only. Histologically, it is characterized by the lack of infiltration of the surrounding tissues. Endometrioid stromal sarcoma affects the uterine corpus, and rarely, the ovaries, cervix, and vagina. In the uterine corpus it is classified as low grade or high grade endometrial stromal sarcoma. In the remainder of the anatomic sites it is classified as low grade endometrioid stromal sarcoma. Undifferentiated sarcoma was previously also known as high grade endometrial stromal sarcoma. In 2014, high grade endometrial stromal sarcoma was reclassified and is currently considered a distinct and rare neoplasm that affects the uterine corpus only. It appears to have a prognosis that falls between low grade endometrial stromal sarcoma and undifferentiated sarcoma. The latter affects the uterine corpus and rarely the remainder of the anatomic sites. 